\title{
1 Route following by one-eyed ants suggests a revised model of normal route following
}

2 Joseph L. Woodgate ${ }^{1,2}$, Craig Perl $^{3,4}$, Thomas S. Collett ${ }^{3}$

3 1. Department of Biological and Experimental Psychology, School of Biological and Chemical Sciences,

4 Queen Mary University of London, Mile End Road, London, E1 4NS, UK.

5 2. Rothamsted Research, West Common, Harpenden, Hertfordshire, AL5 2JQ, UK

6 3. School of Life Sciences, University of Sussex, Brighton, BN19QG, UK

7 4. (current affiliation) Department of Zoology: Functional Morphology, Stockholm University, Svante

8 Arrhenius väg 18b, 106 91, Stockholm, Sweden

\section{Summary}

The prevailing account of visually controlled routes is that an ant learns views as it follows a route, while guided by other path-setting mechanisms. Once a set of route views is memorised,

12 the insect follows the route by turning and moving forwards when the view on the retina

13 matches a stored view. We have engineered a situation in which this account cannot suffice in

14 order to discover whether there may be additional components to the performance of routes.

15 One-eyed wood ants were trained to navigate a short route in the laboratory guided by a single

16 black, vertical bar placed in the blinded visual field. Ants thus had to turn away from the route

17 to see the bar. They often turned to look at or beyond the bar and then turned to face in the

18 direction of the goal. Tests in which the bar was shifted to be more peripheral or more frontal

19 than in training produced a corresponding change in the ants' paths, demonstrating that they

20 were guided by the bar, presumably obtaining information during scanning turns towards the

21 bar. Examination of the endpoints of turns away from the bar suggest that ants use the bar for

22 guidance by learning how large a turn-back is needed to face the goal. We suggest that the

23 ants' zigzag paths are an integral part of visually guided route following. In addition, on some

24 runs in which ants did not take a direct path to the goal, they still turned to face and sometimes

25 approach the goal for a short stretch. This off-route goal facing indicates that they store a

26 vector from start to goal and use path integration to track their position relative to the

27 endpoint of the vector. 
Introduction

Foraging ants, with their faculties intact, readily learn and follow visually guided routes between their nest and a foraging site (Collett et al. 1992, Wehner et al. 1996, Collett, M., 2010, Mangan and Webb 2012, Narendra et al. 2013). They can do so despite a large mismatch between their position on the route and their path integration (PI) state (Kohler and Wehner 2005, Graham and Cheng 2009, Narendra et al., 2013), implying that visual guidance does not require support from $\mathrm{Pl}$, even though the two guidance mechanisms are normally co-active (Collett, M., 2012; Wehner et al., 2016; Hoinville and Wehner 2018). Such experiments and modelling (Baddeley 2012) had suggested that visual route following involves 'alignment image matching' (AIM) (review: Zeil, 2012, Collett et al., 2013). In brief, ants that are guided initially by $\mathrm{PI}$ and by innate responses to obstacles and visual features that they encounter along the way, memorise routes by recording retinotopic views when facing along the route. Thereafter, they can follow the route by turning until they face in the direction that best matches a recorded view from the set of recorded views and then walking forward.

Since wood ants often use the same foraging path when going to and from their nest and on their outward route turn back to face along the path they have just followed, they can use what they have learnt on outward routes to help guide their return (Graham and Collett, 2006; Schwartz et al, 2020). A set of views may thus be associated with a particular motivational context so that an ant interrogates a different set on its approach to food than it does when

47 homing (Harris et al., 2005; Wehner et al., 2005). A recently suggested alternative (Murray et al., 2020 ) is that ants activate both sets for guidance with one as attractive, mediating approach, and the other repulsive, causing avoidance, so improving the precision of route following. To

50 account for the behaviour of wood ants from this perspective (Harris et al. 2005), the

51 motivational context would determine which views are attractive and which repulsive.

Can one-eyed ants follow visually guided routes in the restricted surroundings of a

53 laboratory lacking celestial compass information? In earlier experiments (Buehlmann et al.

54 unpublished data), ants with one eye painted over were trained to find a drop of sucrose at the 55 edge of a circular arena. They were released at the centre of the arena and learnt a straight 
route from there to a point on the circumference. This location was specified by a black vertical

57 bar fixed to the white inner wall of a rotatable cylinder surrounding the arena. When the ants

58 faced along the route, the bar was well outside the visual field of the ants' seeing eye (e.g.,

59 Zollikofer et al., 1995). The cylinder and the position of the food were rotated together from

60 trial to trial to ensure that the black bar was the principal visual cue and also to remove any

61 reliance on a magnetic compass (Çamlitepe and Stradling,1995). Ants with their left eye capped

62 were approximately normal in route learning when the bar was on the right side of the route

63 going from the centre to the periphery of the arena, but struggled when the shape was on the

64 left side (Buehlmann et al. unpublished data). From the perspective of image alignment, this

65 failure is not surprising. To the one-eyed ant, the view along the desired route is of an empty

66 cylinder (Fig 1A) and the ant can fulfil this condition by picking from a wide swath of possible

67 paths. One possible solution to the problem is to walk sideways while facing the cue (Fig 1B) - a

68 strategy that we have not seen the ants adopt
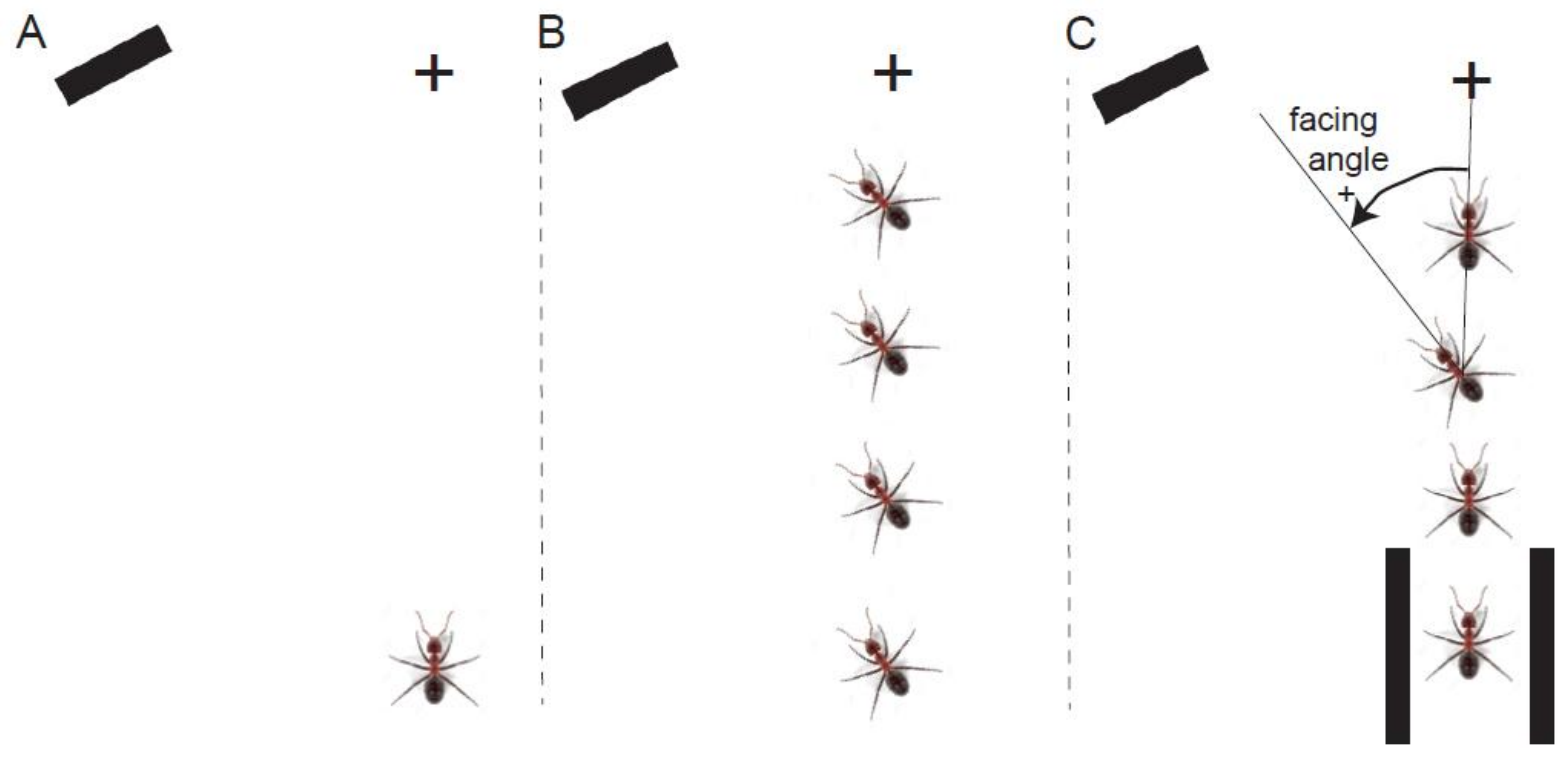

Figure 1. Schematic of problem and solution

71 A: Ant with left eye covered has difficulties in reaching its goal when the only directional aid is a black, vertical stripe outside the right eye's visual field. B: Ant could, but does not approach, the goal when moving sideways. $\boldsymbol{C}$ : Ant is aided by mechanical guides forming a channel at the start of the route. It then turns to face towards and then away from the stripe and can reach the food (+). 
In the present study, performed in 2017 , we followed the same procedure except that we

76 added extra information that seemed to help the ants learn a route. Ants were pointed in the

77 right direction at the start of the route by two short parallel strips of wood aligned along the

78 route (Fig. 1C). We examine the possibility that these guides help ants to develop a food vector

79 (Collett et al., 1999; Bolek et al 2012) (i.e. a memory of the distance and direction from the start

80 to the food); and that the ants' routes are to some degree guided by $\mathrm{PI}$, as they follow this

81 vector. Tests with the vertical bar shifted further or closer to the route direction show that the

82 ants also made use of the visual cue.

\section{Results}

\section{Routes of monocular ants}

Although ants were able to learn the route, the paths are much more variable than those of

86 ants with normal vision guided by a similar cue (c.f. Woodgate et al. 2016). The directions of

87 individual paths from the end of the channel to $15 \mathrm{~cm}$ from the food are shown plotted relative

88 to the position of the food during training. If we select all the paths in the training trials before

89 a test (Figure 2A, S1A), the bulk of paths are in a direction that lies within the expected

90 quadrant (circular median: $2.85^{\circ}$; IQR: $-22.35^{\circ}$ to $11.69^{\circ}$ ). This value does not differ significantly

91 from the direction of the food (binomial test, N: 131, P: 0.16; Zar, 1999). The details of the

92 distribution are complex with a peak just to the right of the goal and another ca $20^{\circ}$ to the left

93 of the goal and subsidiary peaks close to the direction of the bar. 


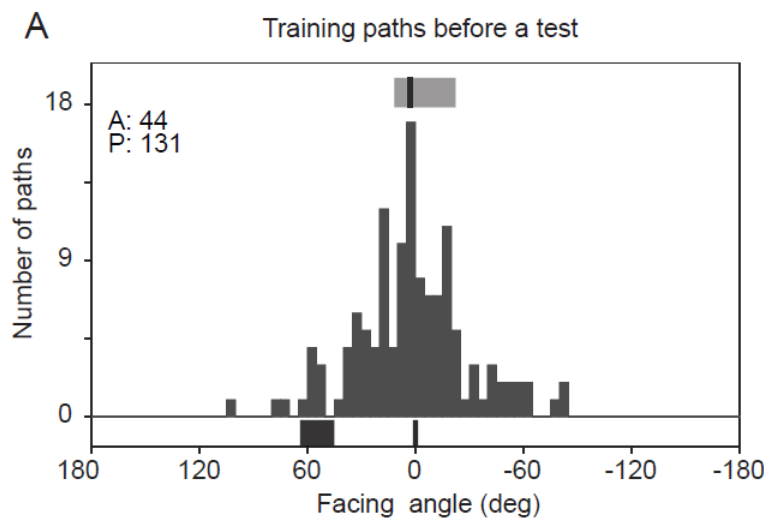

B

Test 1
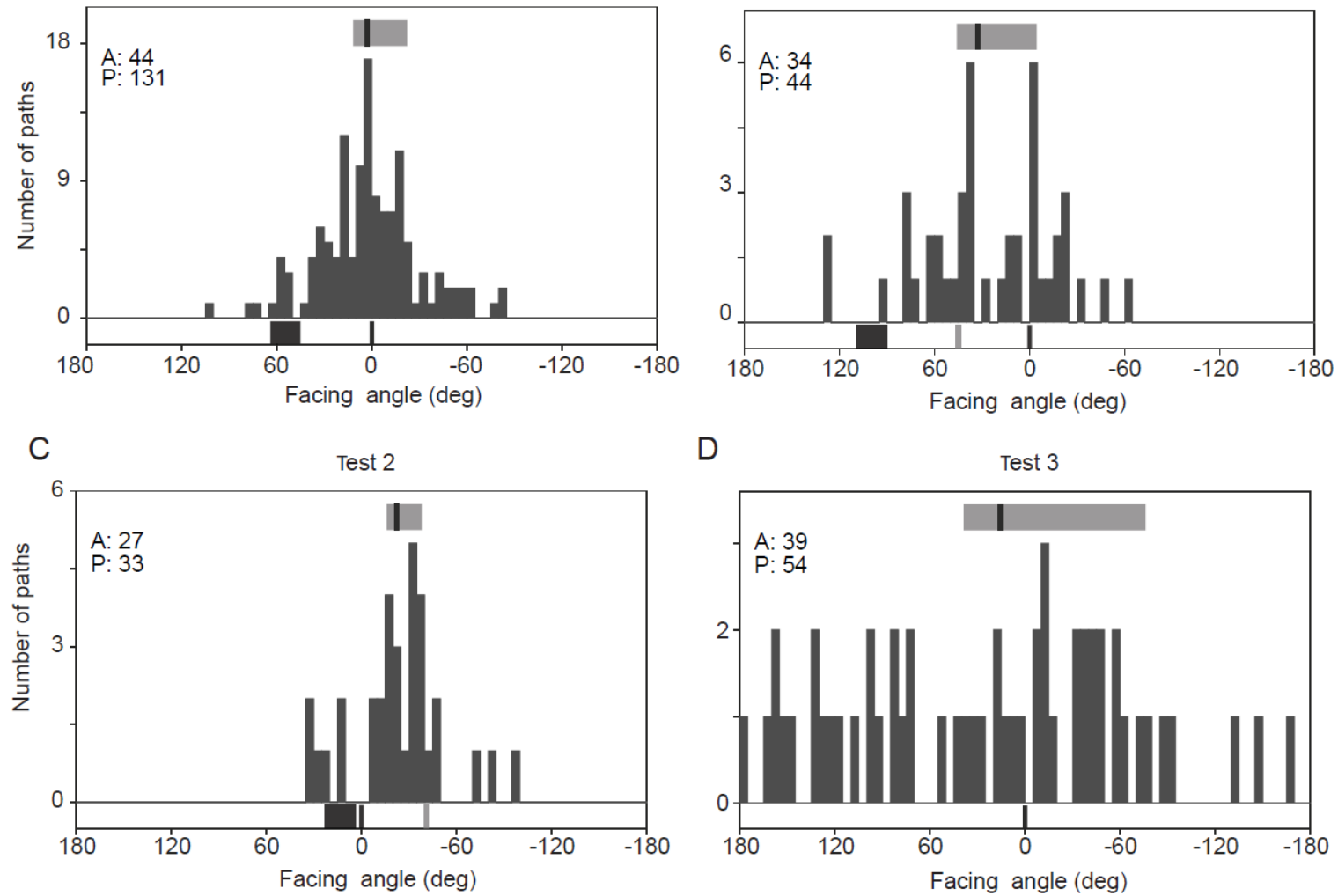

D

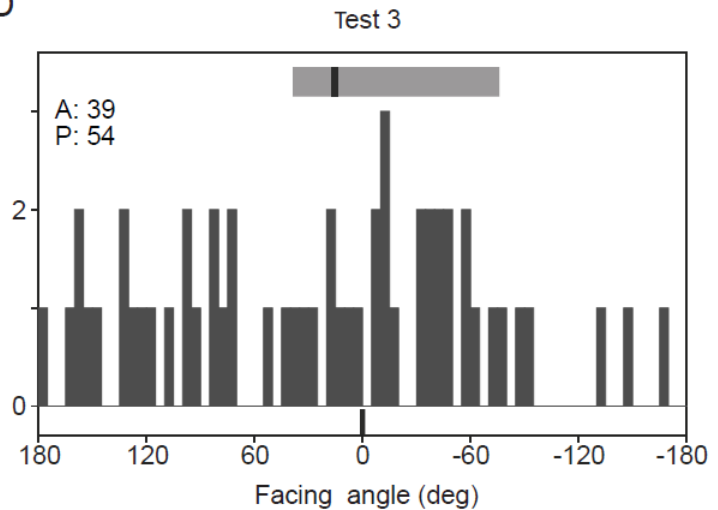

Figure 2. Path histograms

95 Path directions during training and three tests (see Methods). A: histogram showing the overall path directions taken by ants in training trials immediately preceding a test. Bin width is $5^{\circ}$ in all histograms. Zero is the direction of the starting channel points from the centre of the arena (i.e., direction of food during training). In each histogram in this and other figures, A gives the number of ants and $P$ the number of paths; the black line and grey rectangle above the histogram show the median and IQR of the distribution. The black rectangle below the histogram shows the width and position of the visual cue; the black line at $0^{\circ}$ shows the food direction. B: path directions in tests with visual cue shifted left, relative to the starting channel. Black line at $0^{\circ}$ shows the direction in which the starting channel was pointing; the grey line is the predicted goal direction if ants use the visual cue for guidance. $C$ : path directions in tests with the visual cue shifted right, relative to the starting channel. D: path directions in the absence of the visual cue.

Tests in which trained ants were tested with the bar displaced to the left or to the right of

107 its training position suggest that the ants' direction of travel shifts with the displacement. In 108 test 1 , the right edge of the bar is about $90^{\circ}$ from the direction in which the starting channel 109 points (Figure 2 B, Figure S1 B). The number of paths is small $(n=44)$. One of two 'major' peaks $(n=6)$ of the path directions (Figure $3 \mathrm{~B}$ ) is $10^{\circ}$ to the right of the expected goal relative to the 
111 shifted bar (bar-defined predicted heading: $\left.45^{\circ}\right)$. Another peak $(n=6)$ is in the channel direction

112 (channel-defined predicted heading: $0^{\circ}$ ). The circular median path direction (plotted relative to

113 the channel-defined prediction; median $32.41^{\circ}$; IQR $-4.55^{\circ}$ to $45.92^{\circ}$ ) differs significantly from

114 the bar-defined goal (a), but does not differ from the direction of the channel (b) (binomial test,

115 a: $N=44, P: 0.010 ; b: N=44, P: 0.096)$. A comparison of path directions shows that the test 1

116 circular path median differed significantly from the median of training paths both when

117 measured relative to the bar-defined goal direction (a) and relative to the channel direction (b)

118 (Common median test, a: $\mathrm{N}=44,131$, test statistic=10.11, $\mathrm{P}=0.001 ; \mathrm{b}: \mathrm{N}=44,131$, test

119 statistic=4.19, $\mathrm{P}=0.041)$.

In a second test, the bar was moved to the right with its right edge almost in line with the

121 channel. In this case, the paths shifted to the right of the training path (Figure 2 C, Figure S1 C).

122 The peak of the distribution of path directions relative to the food lay between the training

123 direction and the goal relative to the shifted bar (circular median $-22.41^{\circ} ; \mathrm{IQR}=-38.08^{\circ}$ to -

$12415.98^{\circ}, \mathrm{N}: 33$ ). This median differs from both the bar-defined goal (a, bar-defined predicted

125 heading: $-41.2^{\circ}$ ) and from the channel direction (b, channel-defined predicted heading: $0^{\circ}$ )

126 (binomial test, a: N: 33, P: 0.0003 ; b: N: 33, P: 0.0003) The circular median of test 2 differs from

127 the training path median, whether it is measured relative to the bar-defined goal direction (a)

128 or the channel direction (b) (Common median test, a: $\mathrm{N}=33,131$, test statistic=10.96, $\mathrm{P}=0.0009$;

129 b: $N=33,131$, test statistic $=16.73, P<0.0001)$.

In the last test the bar was removed (Figure $2 \mathrm{D}$, Figure S1 D). The median direction is

$13115.54^{\circ}\left(\mathrm{IQR}=-75.87^{\circ}\right.$ to $\left.38.74^{\circ}, \mathrm{N} 54\right)$ and does not differ significantly from the channel

132 direction (binomial test, N: 54, P: 0.497). The dispersion of paths around the median was

133 broader than in training (circular Watson $U^{2}$ test, $U 1.133 ; P<0.01 ; N: 54,131$ ). Nonetheless, the

134 data are not uniformly distributed around the entire circle (Rayleigh test for uniformity: Z: 3.16,

135 P: 0.042).

We conclude from these results that the ants' path directions are influenced by the

137 position of the bar, but that there are additional guidance mechanisms at play, such as a food

138 vector. 
Individual paths

That the multiple peaks in the path direction data may be the consequences of following

141 different navigational mechanisms is supported by the patterns seen in individual paths.

142 Examples of test paths (Figure $3 \mathrm{C}-\mathrm{F}$ ) indicate that ants can be guided by the bar, but also that

143 guidance along the bar defined route can be accomplished with very few frontal views of the 144 bar (Figure 3C). Training routes with few frontal views of the bar (Figure 3A) could mean that

145 ants, guided idiothetically, without compass information, follow their starting directions to the

146 food (e.g. Lent et al., 2009). Ants may also be guided idiothetically by PI, as in Drosophila

147 returning to a drop of sucrose that they have sampled (Kim and Dickinson, 2017).

Suggestive evidence for a food vector monitored through PI comes from examples of ants

149 that leave the direct route to the goal and turn and travel towards the food when they are 150 some distance from the trained path. Deviations from the trained path happens when ants

151 head towards the bar (Figure 4 A-C, G-J) or travel well to the right of the direct route from the 152 centre to the food (Figure $4 \mathrm{D}-\mathrm{F}$ ). These deviant paths can be interrupted by the ant turning 153 towards the goal and moving a short (e.g. Figure 4, F, G) or a longer distance (e.g. Figure 4 E, I)

154 towards it. When the distance towards the goal is longer, and is not just a brief interruption to a 155 path elsewhere (Fig. $4 \mathrm{D}, \mathrm{E}, \mathrm{H}$ ), facing the goal tends to occur at the peaks and troughs of a 156 zigzag approach, as it does in binocular ants (Lent et al., 2013).

157 The examples mentioned so far come from training trials with a drop of sucrose at the goal. 158 Could the ants obtain guidance cues from the sucrose, itself? During experiments over many 159 years in this set up, ants never seem to notice the food until they have almost reached it. These 160 concerns do not arise during tests in which food is always absent. Ants continue to face the 161 channel-defined goal when approaching the bar in test 1 (Figure $4 \mathrm{~J}-\mathrm{L}$ ). 

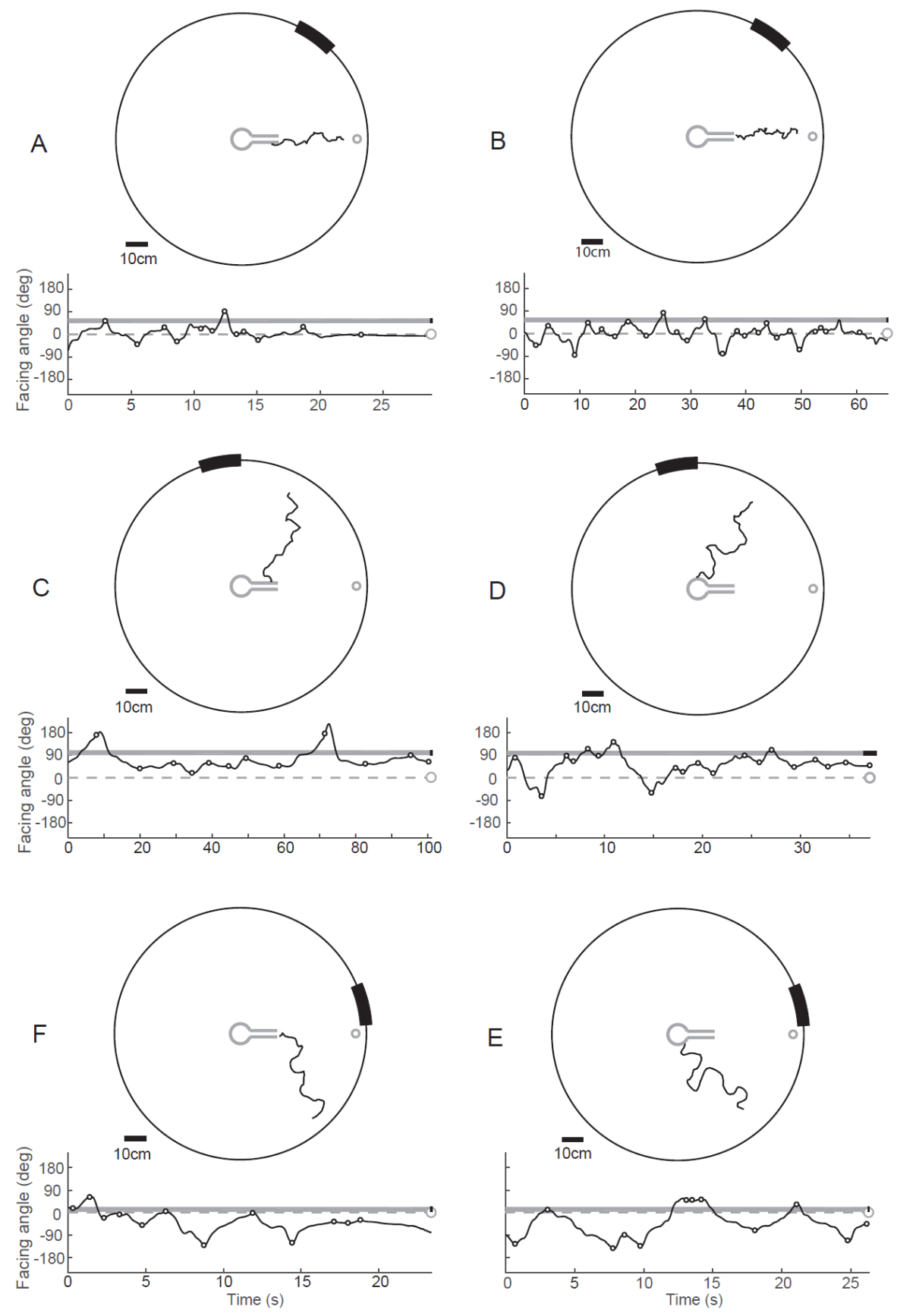

Figure 3. Individual examples of ants' trajectories during training and tests

163 A, B: Training paths. Each panel shows the path of a different ant. Top: path of each ant, seen from above, as it moves from the enclosure and guiding channel in the centre of the arena towards the food position $\left(0^{\circ}\right) 55 \mathrm{~cm}$ away. Black bar on circumference of arena is the projection of the vertical bar that is fixed to the surrounding $180 \mathrm{~cm}$ diameter cylinder. Bottom: Ants' facing angle relative to the food position (+ve angles counterclockwise) are plotted against time from start of the illustrated trajectory. Gray bar shows angular position of bar as measured from arena centre. Circles mark extracted turn extrema. C, D: Test with bar shifted more peripherally relative to the direction of the starting channel (no food present). Other details as in A. E, F: Test with bar shifted centrally relative to starting channel. 
171 Facing and moving towards the goal can most easily be explained by supposing that ants

172 learn a vector that goes from the centre of the arena to the goal and rely on PI to monitor their

173 progress along the vector and to generate a home vector, whatever path they take (cf.

174 Fernandes et al., 2015). The examples in Figure 4 also show how readily ants switch between

175 following PI and controlling their path with a visual cue.

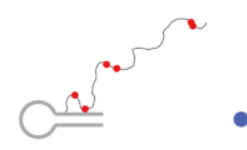

$\mathrm{D}$
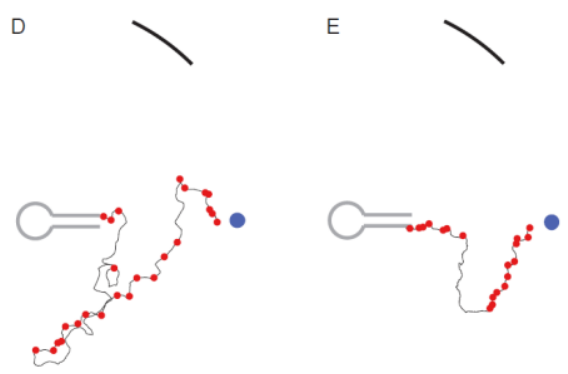

$\mathrm{H}$

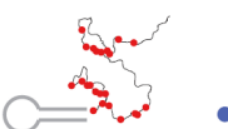

$E$
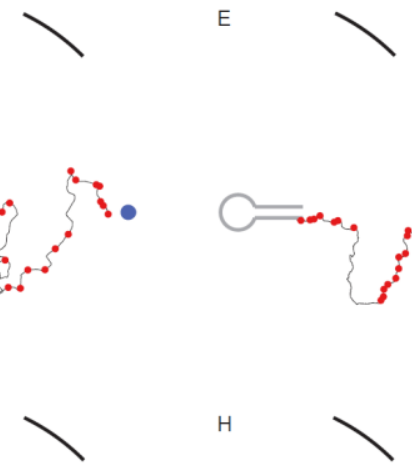
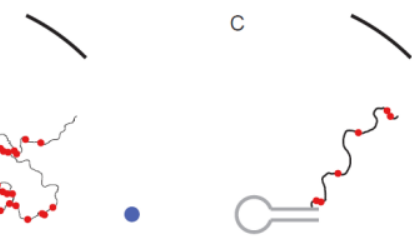

$\mathrm{F}$

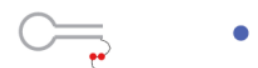

I
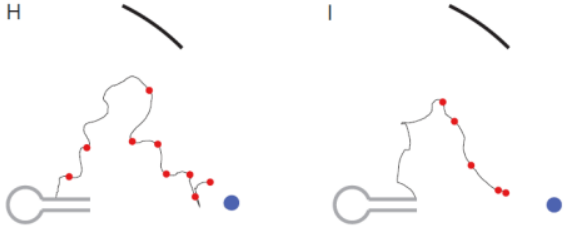
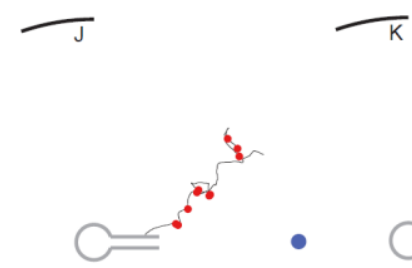

$50 \mathrm{~cm}$
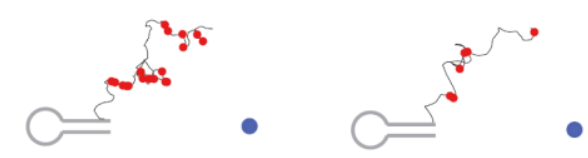

\section{Figure 4. Suggestive evidence that ants can be guided by path integration to the food position}

177 Example paths of ants that deviated far from the direct path to the bar defined goal. Points or path segments in which the ants face the goal within $\pm 10^{\circ}$ are marked with red dots. Panels $\boldsymbol{D}, \boldsymbol{E}, \boldsymbol{H}$ and $\boldsymbol{I}$ show ants that stray far from the direct route between the start and the food, but later make move directly toward the goal, suggestive of the action of a food vector monitored by PI. Other panels show shorter periods of movement in the goal direction and illustrate the range of circumstances under which goal facing occurs. Panels $\mathbf{J}, \boldsymbol{K}$ and $\boldsymbol{L}$ show similar goal-facing in tests in which the position of the visual cue has changed, demonstrating that this behaviour cannot be entirely visually mediated. 


\section{Facing directions at the end points of turns}

185

186

187

188

189

190

191

192

193

194

The distributions of path directions (Figure 2) indicate that the bar has a major influence on the ants' direction of travel. More detail comes from examining separately the endpoints of the left and right turns that ants make during their paths. We extracted two kinds of turn endpoints from the data: i) the maxima and minima in plots of the ants' facing angles (e.g. Figure 3B); ii) turns that end in a plateau, where the ant keeps its facing direction steady for at least $0.2 \mathrm{~s}$ (e.g. Figure $3 \mathrm{~A}$ ) and combined the two kinds of endpoints in all the histograms below (Figure 5).

Despite the wide spread of the distributions, the pattern of peaks is informative. In the pretest training trials, the distributions of left turn endpoints have a clear peak associated with the food which is likely to reflect turns generated through PI (Figure 5A). A second peak consists of left turns that end close to the bar. The endpoints of right turns during training are in the food direction and these end points could either be generated through PI or reflect a right turn of a remembered size after facing the bar (Figure 5C).

In test 1 , the endpoints of left turns are shifted to the left in comparison with training, but the details of the pattern are too noisy to be informative (Figure 5B). The distribution of endpoints of right turns has two peaks, similar to the pattern of path directions during test 1 (Figure 5D, c.f. Figure 2B). One peak is in the bar-specified goal direction and a second is in the channel-specified direction. The peak in the bar-specified goal direction is enhanced, when we select just those right turns that occur after viewing the bar (Figure 5F). To do so, we included only endpoints that followed turns ending within $30^{\circ}$ either side of the edges of the bar. The comparison of this plot with the plot of all right turns during test 1 provides our best evidence that ants do learn the amplitude of the turn that they must make to face in the goal direction after viewing the bar. 
Endpoints during left turns
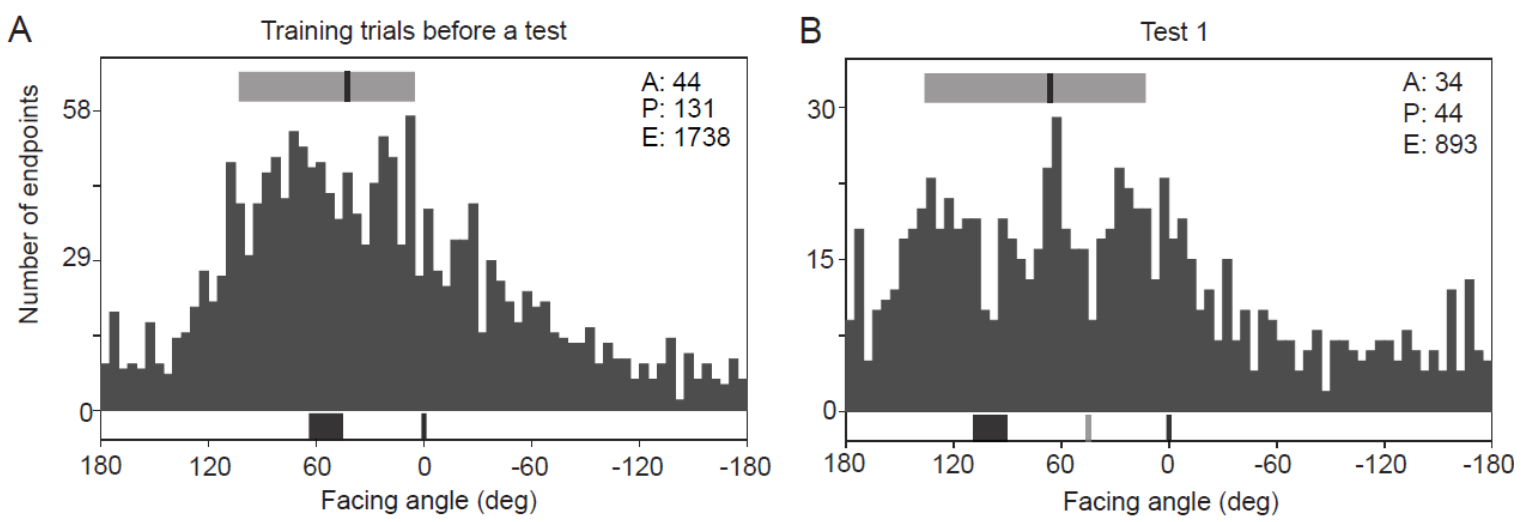

Endpoints during right turns
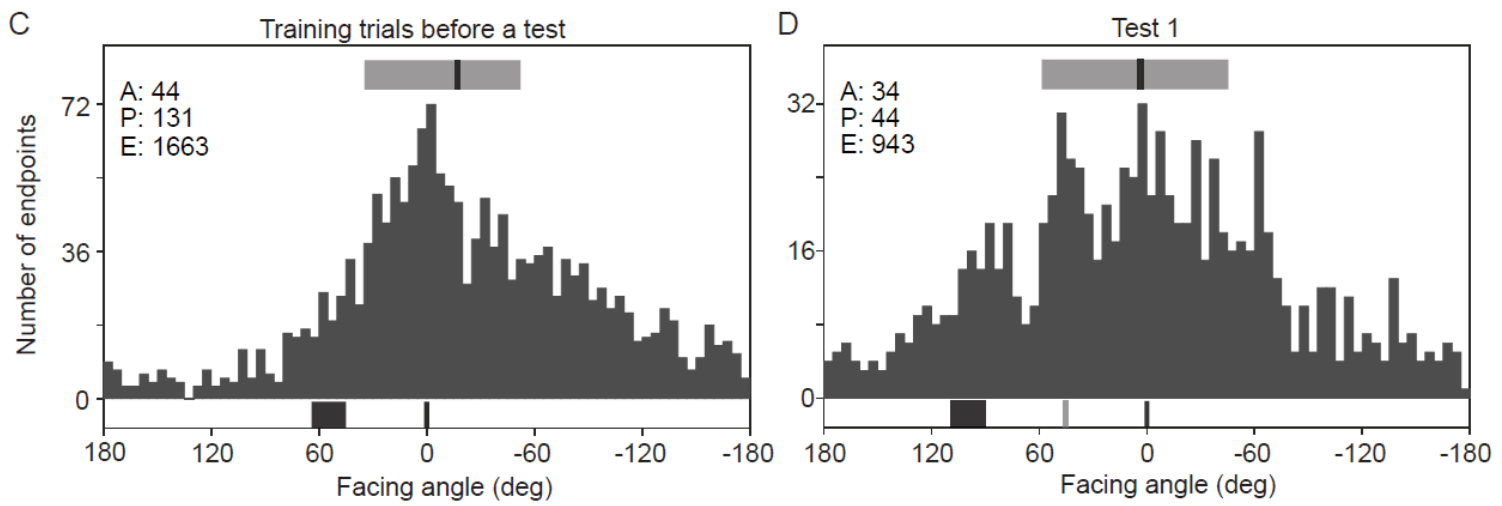

Right turns preceded by left turns that ended within 30 degrees either side of bar
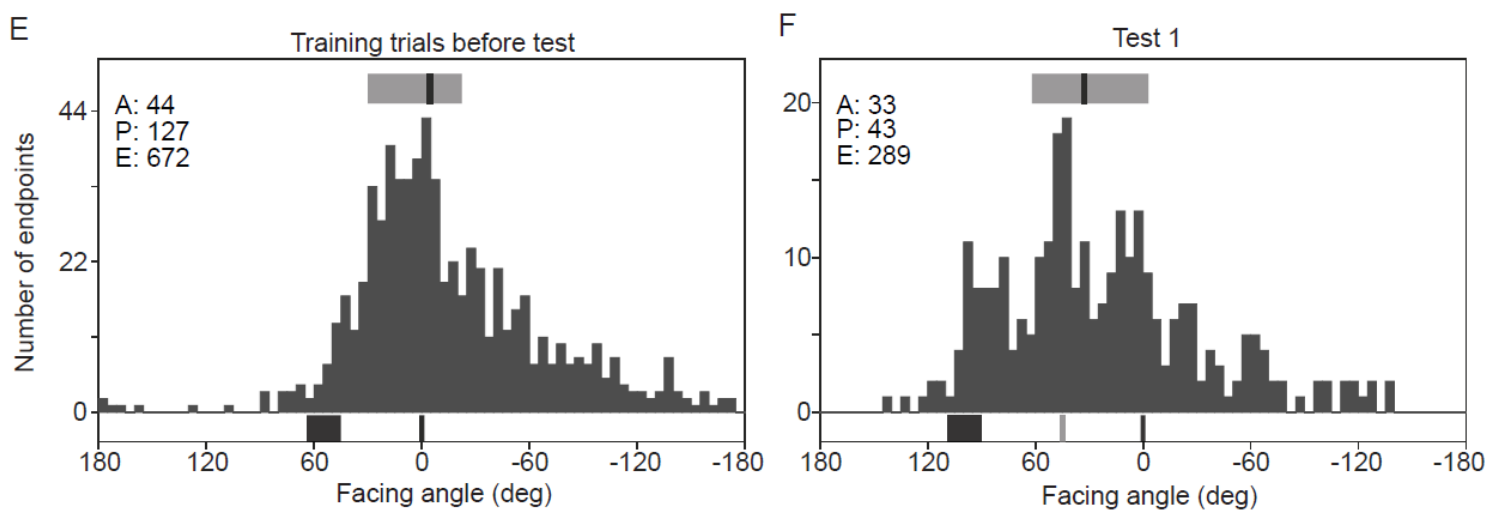

207 Figure 5. Histograms of endpoints of left and right turns.

208 A: All endpoints of left turns during pre-test training trials. B: Endpoints of left turns during Test 1 trials, with visual cue shifted left relative to the starting channel. C, D: Endpoints of right turns in pre-test training and Test 1, respectively. E, F: Endpoints of selected right turns that began $\pm 30^{\circ}$ from the bar edges. In all panels, E denotes number of endpoints. Other details as in Figure 2 
Since we cannot suppose that turn endpoints are independent of each other, we have not

213 attempted statistical analysis. The same pattern is seen when we look at just the median

214 endpoint from each ant path (Figure S2). The small sample number and variability of the data

215 means that the only useful comparison to be made is whether the circular median directions of

216 test 1 (median, relative to the bar-defined predicted goal $=-18.2^{\circ} \mathrm{IQR}=-31.9^{\circ}$ to $14.2^{\circ}, 43$

217 paths) differ from that of test 2 (median, relative to the bar-defined predicted goal $=0.8^{\circ} ; \mathrm{IQR}=$

$218-13.7^{\circ}$ to $13.5^{\circ} ; 32$ paths). We selected all the median endpoints from ants that undertook both

219 tests and aligned the distributions on the bar-defined goals. A common median test

220 demonstrated that the two distributions differed significantly (Common median test, N: 19, 19,

221 test statistic: 5.157, P: 0.023, Fisher, 1995). We conclude that the endpoints of right turns

222 cannot be driven by the position of the visual cue alone but must also be influenced by the

223 mismatch between the bar and channel positions.

Right turn endpoints during test 2 peak at a position that falls between the bar-specified

225 and channel-specified directions. This compromise may be another example of ants averaging

226 directions of the outputs of independent navigational strategies (visual guidance and PI or

227 idiothetic guidance) when the directions of the two outputs are relatively close (Collett, 2012;

228 Wehner et al., 2016; Hoinville and Wehner, 2018).

\section{Binocular ants}

We have re-analysed an earlier study on binocular ants to see whether the findings on one-

231 eyed ants apply to normal route following. Wood ants (Woodgate et al 2016) were trained in

232 the same apparatus to approach a goal with the direction set by a single rectangle placed to the

233 left of the goal direction and tested with wider rectangles. The major result of various tests was

234 that ants seemed to learn their route relative to the centre of mass of the shape. We now ask

235 whether turn endpoints also suggest that ants learn the goal direction relative to the centre of 236 mass of the training shape and wider test shapes. 
Training rectangle

A

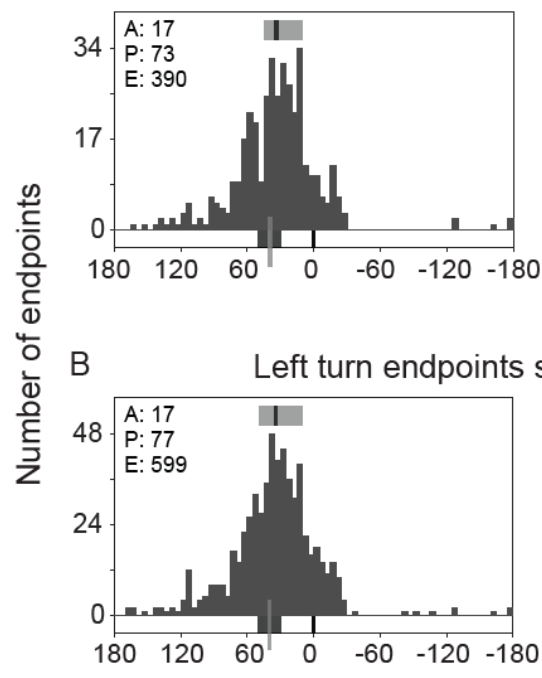

Tests with wider rectangles
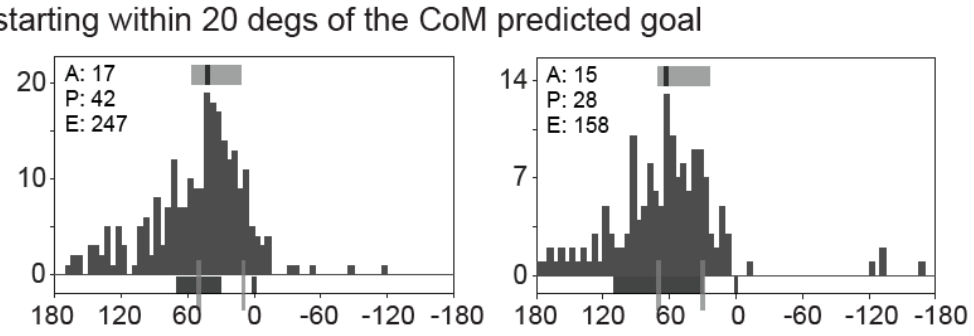

C

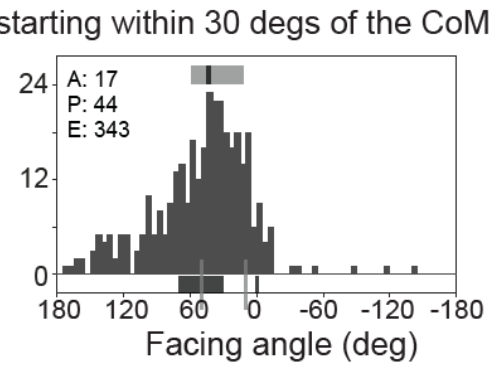

predicted goal

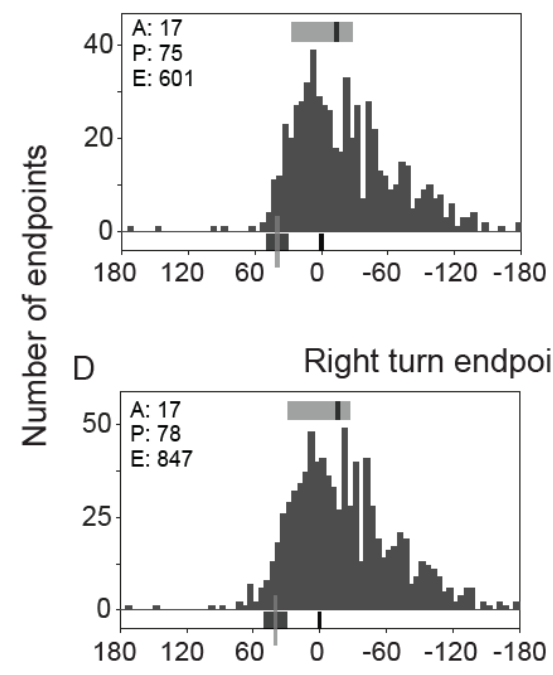

Right turn endpoints starting within 20 degs of the pattern CoM

Figure 6. Histograms of endpoints of binocular wood ants during selected left and right turns.
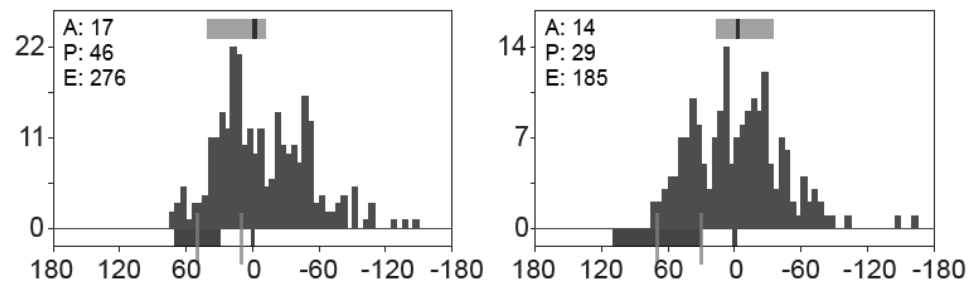

Endpoints of turns in pre-test training trials, tests 1 and tests 2, in which rectangles were increasingly wide. A. Left turns with their starting points within $\pm 20^{\circ}$ of the predicted facing angle of the goal relative to the centre of mass (COM) of each rectangle. Black lines below the histograms at $0^{\circ}$ show the predicted goal direction during training. This direction is also the predicted direction were ants to match the right edge of the rectangle to its position in training. Grey lines below the histograms mark the CoM of the visual cue and the predicted goal direction, were ants to match the CoM of the rectangle to its position in training. Other details as in Figures 2 and 5. B. Left turns with starting points within $\pm 30^{\circ}$ of the predicted facing angle of the goal relative to the CoM of each rectangle. $C$. Right turns with starting points within $\pm 20^{\circ}$ of the CoM of each rectangle. D. Right turns with starting points within $\pm 30^{\circ}$ of the CoM of each rectangle. 
We extracted the endpoints of selected turns during training paths preceding a test and during tests (Figure 6). The selected endpoints of left turns were those that began within $\pm 20^{\circ}$

250 or $\pm 30^{\circ}$ of the predicted goal direction from the midpoint of the width of the training rectangle

251 and wider test rectangles. Selected right turns began within $\pm 20^{\circ}$ or $\pm 30^{\circ}$ of the midpoint of

252 these rectangles. As with monocular ants, the distribution of endpoints is complex and multi

253 peaked. Major peaks of left turns occur a little to the right of the midpoint of the training and

254 wider rectangles and peaks of right turns are a little to the left of the goal direction relative to

255 the midpoint of the rectangle. Given the complexity of the data, we cannot say more than the

256 data are consistent with the importance of the midpoint of the rectangles as one factor

257 influencing the pattern of endpoints and the turns that the ants make.

258 An earlier study of ants approaching visual features aligned with the route found that

259 facing along the route direction tended to occur at the apices of the ants' zigzag path (Lent et al.

260 2013). Examples of paths from (Woodgate et al 2016) show that moments when the ants face

261 the goal head on coincide closely with the peaks and troughs of the ants' paths (Figure 7). The 262 coincidences occur even when the zigzags are irregular. 

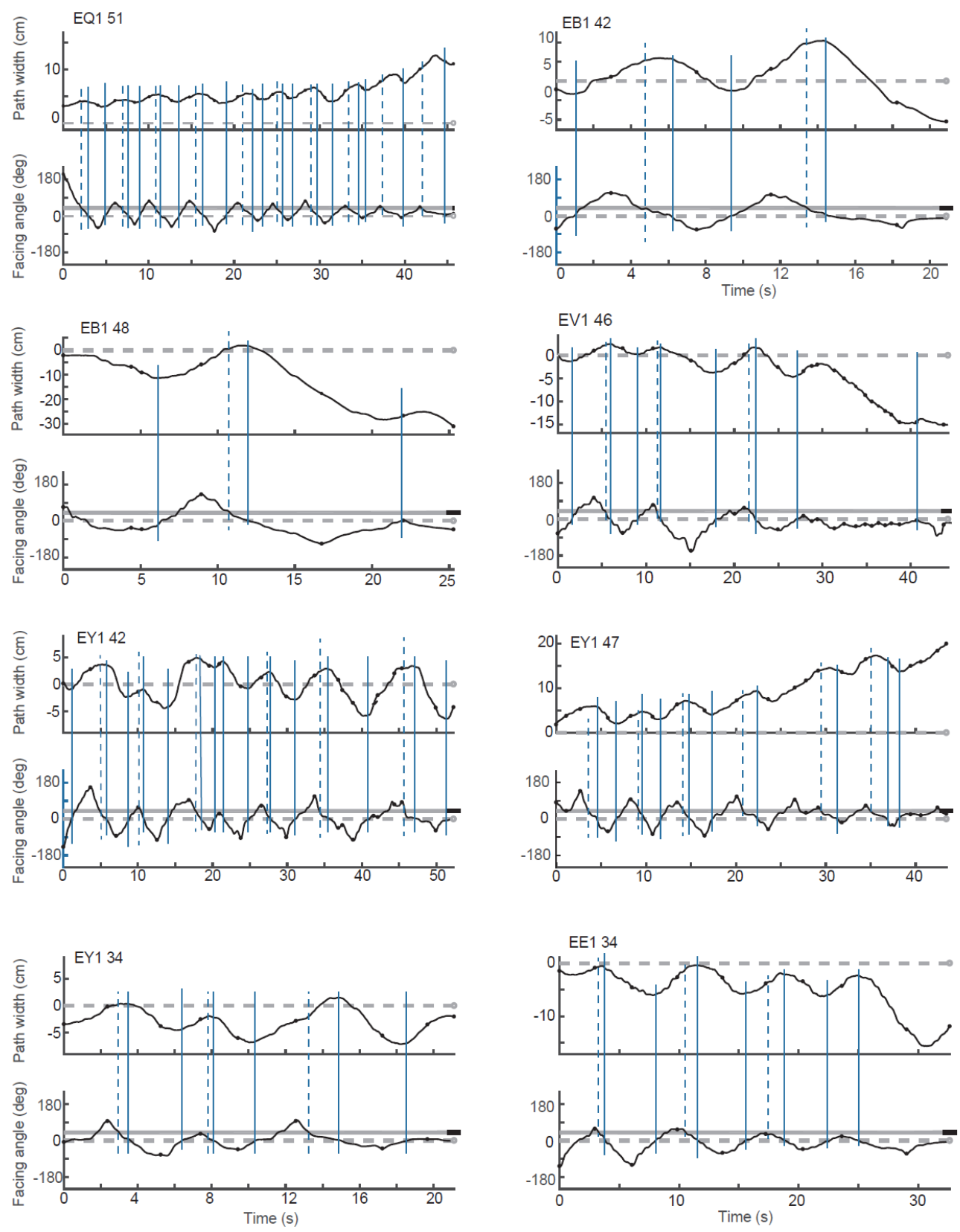

Figure 7. Examples of zigzag paths in binocular wood ants.

Each panel gives one path. Top trace: ant's position along its path plotted against time. Horizontal grey dashed line shows the direct route from the starting point to the food. Bottom trace shows ants' facing angle relative to food. Horizontal dashed grey line shows when facing angle is directly towards the food; grey solid line represents the angular position of the bar as measured from the centre of the arena. Continuous vertical blue lines highlight points at which the ant is facing directly toward the food. These lines tend to intersect the peaks and troughs of the ant's $Y$ position irrespective of the spatial or temporal regularity of the zigzag. Dotted blue vertical lines highlight points at which the ants faced toward the bar during turns in the direction of the food and show the variability of the interval between the ant facing

272 the bar and facing the food. 


\section{Discussion}

274

Alignment image matching, the prevailing account of visually guided navigation in insects,

275 suggests that an animal will turn until its current view matches a previously learned view and

276 then move in that direction (Baddeley et al., 2012). In our study, that process could not operate

277 because one eye was painted over and the only visual cue was on the blind side of the ant.

278 Consequently, when the ant faced in the correct direction, it saw no more than the white arena

279 wall. Ants still learnt routes to a feeder and, if the visual cue was shifted in tests, the ant's paths were deflected in the appropriate direction, demonstrating that the ants' movements were

281 guided by the visual cue.

A wood ant's path tends to oscillate with the desired goal direction reached at the peaks and troughs of the oscillations or zigzags (Lent et al. 2013). This is a common pattern in insect locomotion. It is of clear benefit to insects that move in an oscillating path to follow an odour trail (Hangartner, 1968; Cardé and Willis, 2008; Wllis et al, 2013; Namiki and Kanzaki, 2016). When one-eyed ants zigzagged in the current experiments, they would see the bar during turns to the left, giving them an opportunity to acquire information to guide their subsequent routes. We suggest that, during route learning, the right turns after turning to face the visual cue were guided by path integration so that turns ended with ants facing towards the goal. This turn size can then be learned and later applied when following an acquired route. By this means, visual

291 scanning can support AIM and make it possible to navigate in surroundings with sparse visual 292 information.

The coincidence of facing the along the goal direction with the apices of the zigzag, even 294 when the zigzag is irregular suggests that turns in the goal direction help induce a reversal in 295 the ants' direction of rotation. Because the ants often sustain their direction towards the goal, 296 the influence of goal facing on controlling the direction of rotation seems to be more of a slowly 297 acting nudge than a kick.

298 One puzzle that the data present is that although ants seem able to reach the goal by 299 following a food vector without needing the bar, they mostly did not travel in the food direction 
300 in tests with the bar absent (Figure 2D). A possible explanation comes from previous work on

301 the expression of home and goal vectors that suggests that the vectors are not fully expressed if

302 the surroundings in which they are tested differs markedly from the ant's accustomed

303 surroundings (Cheung et al., 2012). The bar may thus provide a contextual, scene-setting cue as

304 well as a cue for guidance. Another possibility is the lack of optic flow that the bar normally

305 provides.

While AIM as originally conceived is likely to be mediated by the mushroom body (Ardin et

307 al, 2016, Buehlmann et al., 2020; Kamhi et al., 2020) that signals an attractive or aversive

308 direction of travel (Aso et al., 2012), controlled turns to or from specific visual features are

309 probably mediated by visuomotor processing in the central complex (CX) (Collett and Collett,

310 2018). Visual learning of pattern orientation and elevation was some time ago shown to involve

311 ring cells in the ellipsoid body (EB) and cells in the fan-shaped body (Liu et al., 2006; Pan et al.,

312 2009). The experimental technique of these experiments prevented testing whether the

313 azimuth of a visual stimulus is also memorised within these structures.

314 Current research on the Drosophila CX has resolved this question in wonderful detail. First,

315 it was found that a fly's heading direction is correlated with the position of a single 'bump' of

316 electrical activity within the doughnut shaped EB, and that the position of the bump can be

317 locked to a compass direction or to a visual stimulus (Seelig and Jayaraman, 2015; Turner-Evans,

318 D. 2017; Green et al., 2017, Giraldo et al., 2018).

More recent imaging studies and recordings from ring and EP-G neurons in the EB have

320 uncovered the likely mechanism that underlies how the bump of activity within the EB becomes

321 linked to the direction of a visual cue (Fisher et al., 2019; Kim et al., 2019). Individual ring cells

322 in the EB respond to visual cues in a specific retinal position. When a ring cell is visually

323 activated, it inhibits E-PG cells at all positions within the EB, apart from where the ring cell is

324 itself visually activated. This system is plastic such that E-PG cells responsive at a specific

325 heading become linked to a visual cue in that direction after that cue has remained in a fixed

326 direction for a short time. This arrangement allows a facing direction within a visual scene to be 
327 rapidly associated with a particular compass direction, just as is required for a visually

328 controlled route learnt through path integration.

The turn amplitudes that are likely to be mediated by known CX circuitry are of several

330 kinds. Turns that place a visual stimulus in its expected position, like turns towards and away

331 from the bar, can be understood within the framework of the previous paragraph (Fisher et al.,

332 2019; Kim et al., 2019). Turns in the direction of the home vector are also likely to involve the

$333 \mathrm{CX}$, in this case, interactions between the fan-shaped body, the protocerebral bridge and the EB

334 (Neuser et al. 2008; Stone et al., 2017; Honkanen et al 2019, Le Moël et al., 2019). Lastly, the

335 lateral accessory lobes that contain neurons that mediate the turn of a zigzag path (Namiki et al.

336 2016, Steinbeck et al, 2020) may be responsible for the correlated phasing of goal facing and

337 the peaks and troughs of zigzags (Figure 7).

\section{Methods}

339 Ants

340 Experiments were performed on wood ants from laboratory-maintained colonies collected

341 from Broadstone Warren, East Sussex, UK. The colonies were kept under a 12h:12h light-dark

342 cycle and the colony was sprayed with water daily. Water and sucrose dispensers were always

343 available except during experiments when the colony had limited access to sucrose to

344 encourage enthusiastic foraging. Frozen crickets were supplied several times a week.

345 Before ants were trained their left eye was painted with enamel paint and the integrity of 346 the paint cover checked daily under a binocular microscope. The cover appeared stable and it 347 was very rare to have to repaint.

\section{Experimental set-up}

349 The basic experimental procedures followed those described previously (Lent et al., 350 2013). Individually marked wood ants were trained to go from the centre of a circular platform

351 (radius $60 \mathrm{~cm}$ ) towards a drop of sucrose on a microscope slide placed $55 \mathrm{~cm}$ from its centre. The 352 slide was positioned relative to a single black vertical bar $(50 \mathrm{~cm}$ wide and $85 \mathrm{~cm}$ high) that was 
353 cut from black cotton sheeting and fixed to white netting on the white inner wall of a rotatable

354 cylinder (diameter $3 \mathrm{~m}$, height $1.8 \mathrm{~m}$ ). Seen from the centre of the arena, the right edge of the

355 bar was $45^{\circ}$ to the left of the direction the feeder.

To provide idiothetic cues, a pair of guide sticks ( $3 \mathrm{~mm}$ square cross-section) with a $24 \mathrm{~mm}$ path between them led from a ring $(5 \mathrm{~cm}$ radius and $25 \mathrm{~mm}$ high) surrounding the centre of the arena. In initial training trials, the sticks led most of the way to the food, their length was gradually shortened to $13 \mathrm{~cm} .13 \mathrm{~cm}$ and $10 \mathrm{~cm}$ sticks were used for tests. See Fig. 3 for a top view of the arena the arrangement of sticks, the sucrose and the radial position of the bar.

\section{Experimental procedure}

Ants were given about 25 trials of group training over about 3 days before being trained individually. On each trial, individually marked ants from a cohort of ca. 25, were taken from

364 the nest and separated into groups of between 6 and 8 individuals. One group at a time was 365 placed in the ring, sometimes overlapping with stragglers from a previous group. After the ants 366 had reached the feeder and had started to drink, they were placed in a box with sucrose and 367 drank to completion before being returned to the nest.

For individual training, ants were put singly into a $6.5 \mathrm{~cm}$ diameter, cylindrical holding chamber that lay within the ring. The wall of the holding chamber could be lowered remotely

370 from outside the cylinder to be flush with the arena floor. Once the wall was lowered the ant 371 was free to leave the ring. When the ant had reached the food reward and started to feed, or

372 reached the edge of the arena, the experimenter raised the wall of the holding chamber,

373 entered the cylinder, transferred the ant to a feeding box and placed the next ant in the holding 374 chamber. After the cohort of ants had completed a training trial, the guide sticks, the black bar 375 and the slide with food were rotated to a new position to avoid the ants learning other cues for 376 guidance.

Once individual training began, we recorded the ants' individual paths, starting just before each ant was released from the holding chamber. Each ant's movements were recorded using a

379 tracking video camera (Trackit, SciTrackS GmbH), which gave as output the ant's position on the 
arena and the orientation of its body axis every $20 \mathrm{~ms}$. Each ant was released from the holding chamber and its path recorded until it reached the feeder or the edge of the platform. Once it had started to feed, or if it was lost, it was placed in a feeding box.

After individuals had performed 30 training trials, three different tests were introduced with a varying number of intervening training trials. In test 1 , the right edge of the bar was shifted to $90^{\circ}$ to the left of the direction in which the starting channel was pointing. In a second test the bar was shifted in the opposite direction, $\mathrm{ca} 5^{\circ}$ to the left of the channel direction. In

387 the third test the bar was removed. No sucrose reward was present during tests. Ants were removed from the arena once they reached the edge of the platform and allowed to feed to satiety before being returned to the nest.

\section{Data analysis}

We examined two features of the ants' trajectories: 1 . The direction of an ant's path; 2. The ant's facing directions at the extrema of its turns to the left and the right, measured relative to 393 the position of the food (see Figure 1C).

\section{Path direction}

The direction of the ants' paths was analysed from when an ant left the guide channels,

396 beginning ca. $18 \mathrm{~cm}$ from the centre of the arena, or sooner if ants climbed over the guide strips, 397 until they reached a radial distance of $45 \mathrm{~cm}$ from the centre. The direction over this path398 segment was estimated in two stages. First, the path (consisting of $x-y$ coordinates recorded at $39950 \mathrm{fps}$ ) was divided into successive $1.5 \mathrm{~cm}$ segments, and orthogonal distance regression (Gene 400 et al., 1996) was used to calculate the angle of a best fit line through each segment, minimising 401 the error in both $\mathrm{x}$ and $\mathrm{y}$ dimensions (custom written MATLAB script based an algorithm 402 devised by Eitan, T. [https://stackoverflow.com/questions/12250422/orthogonal-distance403 regression-in-matlab]). The overall direction of the path was then taken to be the vector sum of 404 the directions of all path segments until the ant's path took it $45 \mathrm{~cm}$ from the centre of the 405 arena. 


\section{The amplitude of scans}

407

408

409

410

411

412

413

414

415

416

Plots of facing angles over time tend to have peaks and troughs. The facing angle at a peak or a trough was defined as the most extreme angle to the left or right before a change in the direction of rotation. These extrema were identified using the following procedure: first, to reduce noise in the data, the ant's facing directions throughout each track were smoothed by taking the circular mean facing direction across a 50-frame moving window. Points at which the angular change between consecutive frames reversed direction gave the rough location of inflection points in the track. To pick out the facing direction just prior to a reversal of direction, we used the unsmoothed angle data to find the interval over which the facing angle was more extreme than the facing angle at the inflection point. The mid-point of this interval was taken as the facing angle at the peak or trough.

The algorithm used to extract extrema does not capture all the turn-endpoints that can be seen by eye (e.g. Figure 3), because some turns were too small for the inflection point to show up in the smoothed angle data. We saw no indication of false identification of extrema.

Some turns end in a clear plateau rather than a turn in the opposite direction. When this was the case, the ant's facing angles at these plateaux were added to the extrema data to give what we term turn endpoints. Plateaux were identified through the fulfilment of two criteria: (1) an interval during a track that lasted at least $0.2 \mathrm{~s}$, in which the maximum change in facing angles was less than $3^{\circ} ;(2)$ that the slope of the regression of facing angle against time of a candidate plateau should be less than $1^{\circ} \mathrm{s}^{-1}$. The facing angle of an identified plateau was then defined as the circular mean of all facing angles in the interval.

\section{Exclusion of inconsistent ants}

Some ants were very erratic. Paths of these ants are included in Figure S1 but excluded in further analysis. To remove these ants, we calculated the overall direction of travel of each path from every ant and calculated the resultant vector length of the path directions of each ant across the entire training period. Ants whose resultant vector length $>0.4$ took similar directions, relative to the visual and idiothetic cues, in each trial, despite the rotation of the 
433 experimental arena. Those with a vector length $<0.4$ could not cope with rotation; 5 such ants

434 were removed from the training and test dataset. One of these 5 had taken part in tests 1 and

4352 , and none had participated in test 3.

\section{Descriptive statistics}

437 At the top of each histogram, we show the circular median of the distribution (black line;

438 Otieno and Anderson-Cook, 2003) and the interquartile range (IQR; grey bar; defined for

439 circular data, using the likelihood-based arc method, as the smallest arc of the circle containing

$44050 \%$ of all datapoints, Fisher and Hall, 1989). Rayleigh's tests for non-uniformity confirmed that

441 the ants' path directions and endpoints in training and tests 1 and 2 were not uniformly

442 distributed but showed a tendency to head in one direction (all $\mathrm{P}<0.0001$ ). 


\section{References}

445 Ardin, P., Peng, F., Mangan, M., Lagogiannis, K. and Webb, B. (2016). Using an insect mushroom

446 body circuit to encode route memory in complex natural environments. PLoS Comp. Biol. 12,

$447 \mathrm{e} 1004683$.

448 Aso, Y., Sitaraman, D., Ichinose, T., Kaun, K. R., Vogt, K., Belliart-Guerin, G., Plaçais, P.-Y., Robie,

449 A. A., Yamagata, N. and Schnaitmann, C. (2014). Mushroom body output neurons encode

450 valence and guide memory-based action selection in Drosophila. eLife 3, e04580.

451 Baddeley, B., Graham, P., Husbands, P. and Philippides, A. (2012). A model of ant route

452 navigation driven by scene familiarity. Plos Comp. Biol. 8, e1002336.

453 Bolek, S., Wittlinger, M. and Wolf, H. (2012). Establishing food site vectors in desert

454 ants. J.Exp.Biol, 215, 653-656.

455 Buehlmann, C., Wozniak, B., Goulard, R. Webb, B. Graham, P. and Niven, J.E (2020) Mushroom

456 bodies are required for learned visual navigation, but not for innate visual behaviour in ants.

457 Curr. Biol. 30,

458 Çamlitepe, Y. and Stradling, D. J. (1995). Wood ants orient to magnetic fields. Proc R. Soc.B, 261, $459 \quad 37-41$.

460 Cardé R.T. and Willis M.A. 2008 Navigational strategies used by insects to find distant, wind-

461 borne sources of odor. J. Chem. Ecol. 34, 854-866.

462 Cheung, A., Hiby, L. and Narendra, A. (2012). Ant navigation: fractional use of the home 463 vector. PLoS One, 7, e50451.

464 Collett M. 2010 How desert ants use a visual landmark for guidance along a habitual route. Proc. 465 Natl Acad. Sci. USA 107, 11 638-11 643.

466 Collett, M. (2012). How navigational guidance systems are combined in a desert ant. Curr. Biol. $467 \quad 22,927-932$. 
468 Collett, M., Collett, T. S. and Wehner, R. (1999). Calibration of vector navigation in desert ants. 469 Curr. Biol. 9, 1031-1034.

470 Collett, M. and Collett, T.S. (2018). How does the insect central complex use mushroom body 471 output for steering? Curr. Biol. 28, R733-R734.

472 Collett, M., Chittka, L. and Collett, T. S. (2013). Spatial memory in insect navigation. Curr. Biol. $473 \quad 23$, R789-R800.

474 Collett, T. S., Dillmann, E., Giger, A. and Wehner, R. (1992). Visual landmarks and route 475 following in desert ants. .J Comp. Physiol. A, 170, 435-442.

476 Cardé, R. T. and Willis, M. A. (2008). Navigational strategies used by insects to find distant, 477 wind-borne sources of odor. J. Chem. Ecol., 34(7), 854-866.

478 Fernandes, A. S. D., Philippides, A., Collett, T. S. and Niven, J. E. (2015). Acquisition and 479 expression of memories of distance and direction in navigating wood ants. J. Exp. Biol. 218, $480 \quad 3580-3588$.

481 Fisher, N. I. (1995). Statistical analysis of circular data. Cambridge University Press.

482 Fisher, N. I. and Hall, P. (1989). Bootstrap confidence regions for directional data. J.Amer Stat. 483 Assoc. 84, 996-1002.

484 Fisher, Y.E., Lu, J., D’Alessandro, I. and Wilson, R.I. (2019). Sensorimotor experience remaps 485 visual input to a heading-direction network. Nature 576, 121-125.

486 Gene H. Golub and Charles F. Van Loan (1996) "Matrix Computations" (3rd ed.). The Johns 487 Hopkins University Press. pp 596.

488 Giraldo, Y.M., Leitch, K.J., Ros, I.G., Warren, T.L., Weir, P.T. and Dickinson, M.H. (2018). Sun 489 navigation requires compass neurons in Drosophila. Curr. Biol. 28, 2845-2852. 
490 Graham, P. and Cheng, K. (2009). Ants use the panoramic skyline as a visual cue during

491 navigation. Curr. Biol. 19, R935-R937.

492 Graham, P., \& Collett, T. S. (2006). Bi-directional route learning in wood ants. J. Exp. Biol. 209., 493 3677-3684.

494 Green, J., Adachi, A., Shah, K.K., Hirokawa, J.D., Magani, P.S. and Maimon, G. (2017). A neural 495 circuit architecture for angular integration in Drosophila. Nature 546, 101-106.

496 Green, J., Vijayan, V., Mussells Pires, P., Adachi, A. and Maimon, G. (2019). A neural heading 497 estimate is compared with an internal goal to guide oriented navigation. Nat. Neurosci. 22, $498 \quad 1460-1468$.

499 Hangartner, W. 1969 Structure and variability of the individual odor trail in Solenopsis geminata 500 Fabr. (Hymenoptera, Formicidae). Z. Vergl. Physiol. 62,111-120.

501 Harris, R. A., de Ibarra, N. H., Graham, P. and Collett, T. S. (2005). Priming of visual route 502 memories. Nature, 438, 302-302.

503 Hoinville, T. and Wehner, R. (2018). Optimal multiguidance integration in insect navigation. Proc. 504 Natl. Acad. Sci. USA 115, 2824-2829.

505 Honkanen, A., Adden, A., da Silva Freitas, J. and Heinze, S. (2019). The insect central complex 506 and the neural basis of navigational strategies. J. Exp. Biol. 222, jeb188854.

507 Kim, I. S. and Dickinson, M. H. (2017). Idiothetic path integration in the fruit fly Drosophila 508 melanogaster. Curr. Biol. 27, 2227-2238.e3.

509 Kamhi, J.F., Barron, A.B. and Narendra, A. (2020). Vertical lobes of the mushroom bodies are 510 essential for view-based navigation in Australian bull ants. Curr. Biol. 30, 1-6

511 Kim, S.S., Hermundstad, A.M., Romani, S., Abbott, L.F. and Jayaraman, V. (2019). Generation of 512 stable heading representations in diverse visual scenes. Nature 576, 126-131. 
513 Kohler, M. and Wehner, R. (2005). Idiosyncratic route-based memories in desert ants,

514 Melophorus bagoti: How do they interact with path-integration vectors? Neurobiol. Learn.

515 Memory 83, 1-12.

516 Lent, D. D., Graham, P. and Collett, T. S. (2009). A motor component to the memories of

517 habitual foraging routes in wood ants? Curr.Biol. 19,115-121.

518 Lent D.D., Graham P. and Collett T.S. (2013). Phase-dependent visual control of the zigzag paths

519 of navigating wood ants. Curr. Biol. 23, 2393-2399.

520 Liu, G., Seiler, H., Wen, A., Zars, T., Ito, K., Wolf, R., Heisenberg, M. and Liu, L. (2006). Distinct

521 memory traces for two visual features in the Drosophila brain. Nature 439, 551-556.

522 Le Moël, F., Stone, T., Lihoreau, M., Wystrach, A. and Webb, B. (2019). The central complex as a

523 potential substrate for vector based navigation. Front. Psychol. 10,

524 90.doi:10.3389/fpsyg.2019.00690690.

525 Mangan, M. and Webb, B. (2012). Spontaneous formation of multiple routes in individual 526 desert ants (Cataglyphis velox). Behav. Ecol. 23, 944-954.

527 Murray, T., Zoltán, K., Dahmen, H., Le Moël, F., Wystrach, A. and Zeil, J. (2020). The role of

528 attractive and repellent scene memories in ant homing (Myrmecia croslandi). J. Exp. Biol. 223,

529 jeb210021.

530 Namiki, S. and Kanzaki, R. (2016). The neurobiological basis of orientation in insects: insights

531 from the silkmoth mating dance. Curr. Opin. Insect Sci. 15, 16-26.

532 Narendra, A., Gourmaud, S. and Zeil, J. (2013). Mapping the navigational knowledge of

533 individually foraging ants Myrmecia croslandi. Proc. R. Soc. Lond. B 280, 20130683.

534 Neuser, K., Triphan, T., Mronz, M., Poeck, B. and Strauss, R. (2008). Analysis of a spatial 535 orientation memory in Drosophila. Nature 453, 1244-1247. 
536 Otieno, B. and Anderson-Cook, C. M. (2003). A more efficient way of obtaining a unique median 537 estimate for circular data. J. Mod. Appl. Stat. Methods 2, 168-176.

538 Pan, Y., Zhou, Y., Guo, C., Gong, H., Gong, Z. and Liu, L. (2009). Differential roles of the fan539 shaped body and the ellipsoid body in Drosophila visual pattern memory. Learn Mem.16, 289540295.

541 Seelig, J.D. and Jayaraman, V. (2015). Neural dynamics for landmark orientation and angular 542 path integration. Nature 521, 186-191.

543 Stone, T., Webb, B., Adden, A., Weddig, N. B., Honkanen, A., Templin, R., Wcislo, W., Scimeca, L., 544 Warrant, E. and Heinze, S. (2017). An anatomically constrained model for path integration in 545 the bee brain. Curr. Biol. 27,3069-3085.e11.

546 Turner-Evans, D., Wegener, S., Rouault, H., Franconville, R., Wolff, T., Seelig, J.D., Druckmann, S. 547 and Jayaraman, V. (2017). Angular velocity integration in a fly heading circuit. eLife 6, e23496.

548 Webb, B. and Wystrach, A. (2016). Neural mechanisms of insect navigation. Curr. Opin. Insect 549 Sci. 15, 27-39.

550 Wehner, R., Boyer, M., Loertscher, F., Sommer, S. and Menzi, U. (2006). Ant navigation: one551 way routes rather than maps. Curr. Biol. 16, 75-79.

552 Wehner, R., Hoinville, T., Cruse, H. and Cheng, K. (2016). Steering intermediate courses: desert 553 ants combine information from various navigational routines. J. Comp. Physiol. A 202, 459-472.

554 Wehner, R., Michel, B. and Antonsen, P. (1996). Visual navigation in insects: coupling of 555 egocentric and geocentric information. J. Exp. Biol. 199, 129-140.

556 Willis, M. A., Ford, E. A., and Avondet, J. L. (2013). Odor tracking flight of male Manduca sexta 557 moths along plumes of different cross-sectional area. J. Comp. Physiol A, 199, 1015-1036. 
558 Woodgate, J. L., Buehlmann, C. and Collett, T. S. (2016). When navigating wood ants use the

559 centre of mass of a shape to extract directional information from a panoramic skyline. J. Exp.

560 Biol. 219, 1689-1696.

561 Zar, J. H. (1999). Biostatistical analysis. Pearson Education India.

562 Zeil, J. (2012). Visual homing: an insect perspective. Curr. Op. Neurobiol. 22, 285-293.

563 Zollikofer, C. P. E., Wehner, R. and Fukushi, T. (1995). Optical scaling in conspecific Cataglyphis

564 ants. J. Exp. Biol. 198, 1637 
bioRxiv preprint doi: https://doi org/10.1101/2020 $08.21261354^{*}$ this version posted August 24,2020 . The copyright holder for this preprint (which was not certified by peer review) is the author/funder, who has granted bioRxiv a license to display the preprint in perpetuity. It is made available under aCC-BY-NC-ND 4.0 International license.
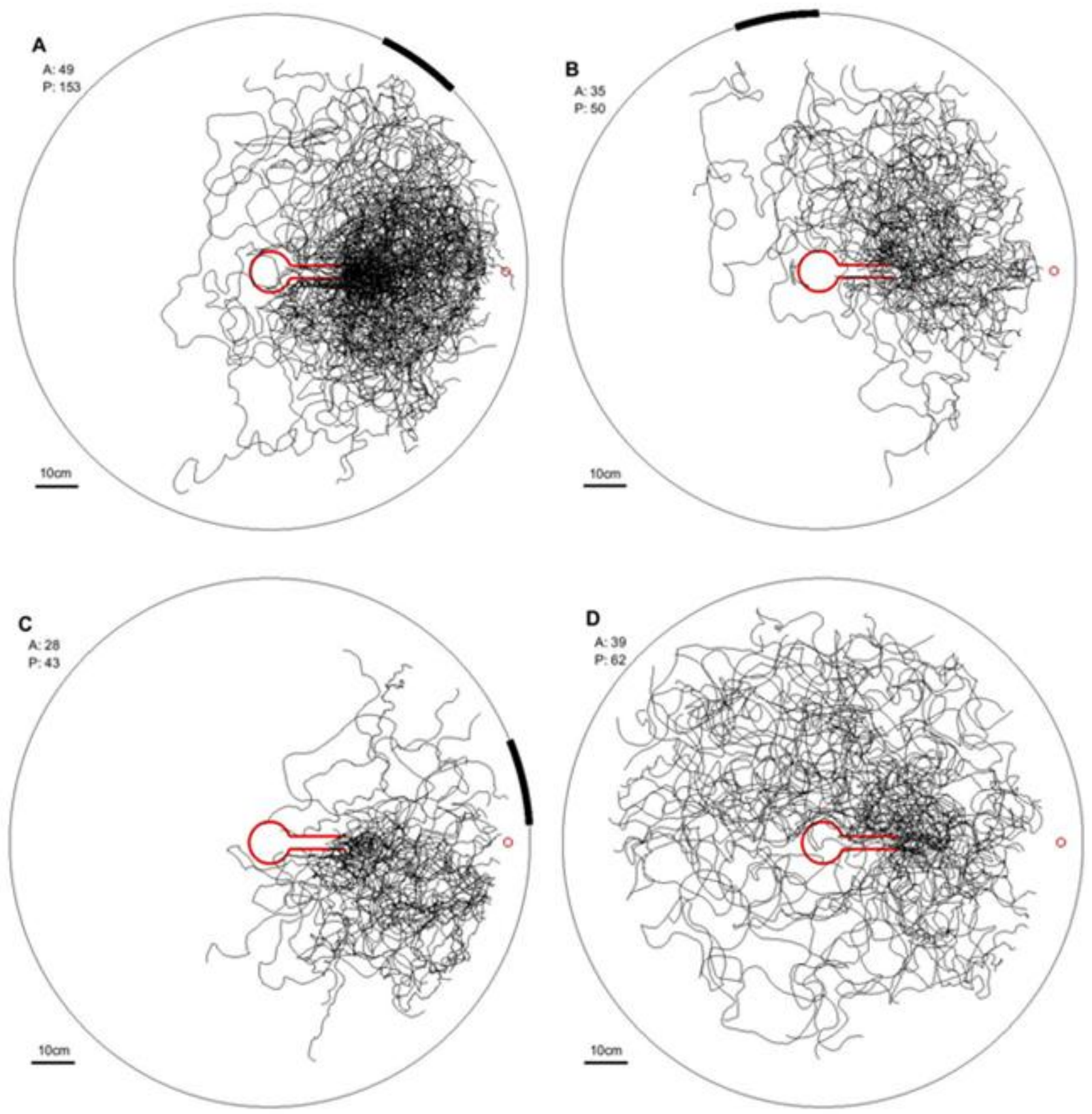

567 Figure S1. Superimposition of ants' paths during training conditions and tests.

568 All paths in training trials just before a test and in tests 1-3 are shown superimposed without any filtering

569 for consistency (See 'Exclusion of inconsistent ants' in Methods). A. paths recorded in training trials B.

570 Paths in test 1 with bar shifted away from food. C. Paths in test 2 with bar shifted towards the food. $\boldsymbol{D}$.

571 Paths in test 3 with the bar removed. In each panel, A gives the number of ants and $P$ the number of

572 paths. 


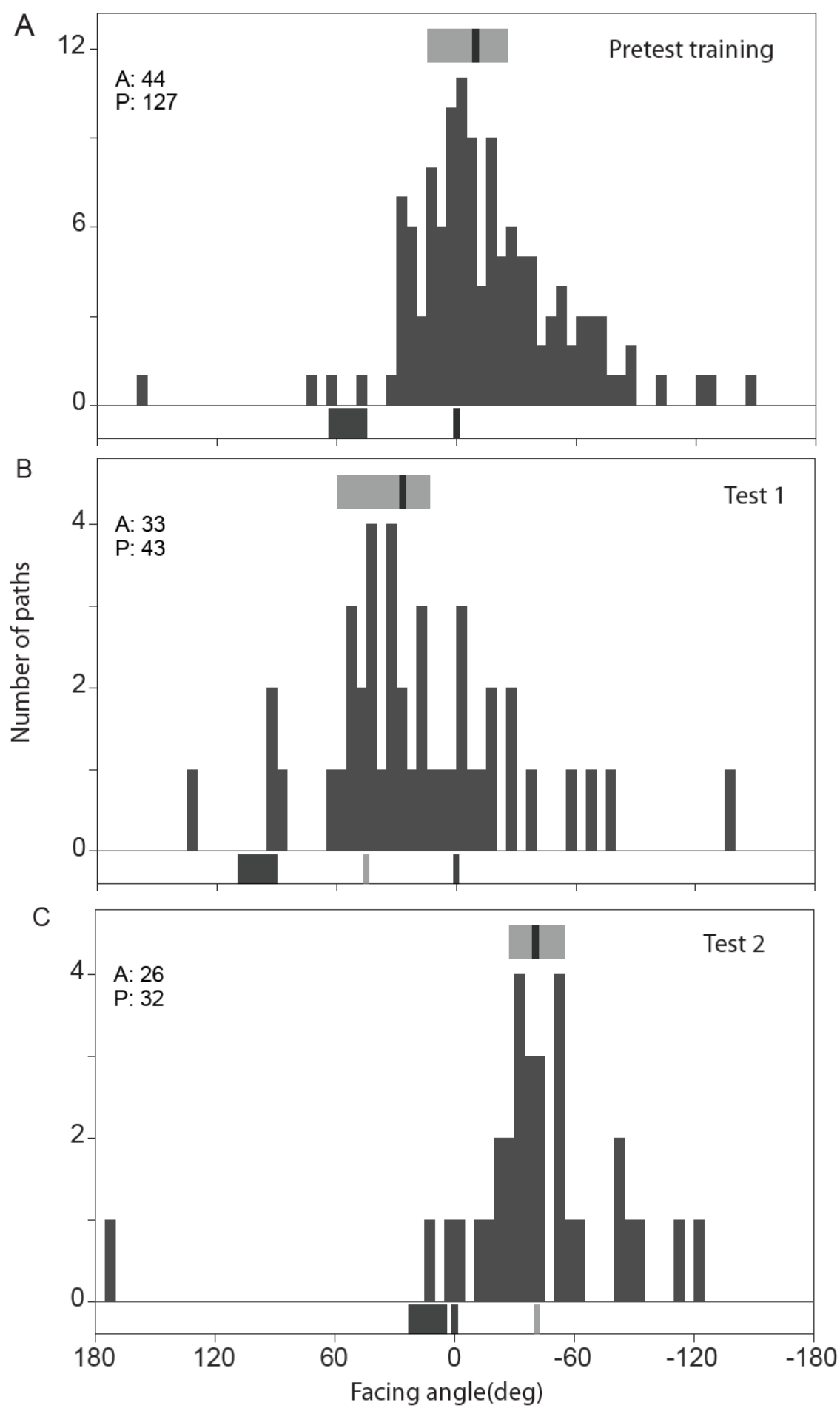

$574 \quad$ Figure S2. Replot of endpoints of selected right turns as in Figure 5.

575 A: Histogram showing, for every pre-test training path, the median of the endpoints of selected right turns within the path. Within each path, selected turns began $\pm 30^{\circ}$ from the bar edges. Each data point represents one path. The pattern of peaks resembles that of Figure 5 B: Median endpoints of selected turns in test 1 trials, with the visual cue shifted left, relative to the starting channel. C: Median endpoints of selected turns in test 2 trials, with the visual cue shifted right, relative to the starting channel. 